IPPARTAMENTO DF ANATOMIA DESCRITIYA DOS ANIMAIS DOMESTICUS Diretor: Prof. Dr. M. Barrus Frhart

DEPARIAMENTO DE TOOTLC,NIA

Diretor: Prof. Dr. Joz̄o Soares Veigr

\title{
DETERMINAÇÃO DA IDADE DO PURO SANGUE DE CARREIRA NA ÉPOCA DAS MUDAS(")
}

\author{
DHFFRMINATION OF TIE AGE IN THE THOROUGHBRED DURING \\ THE REPLACEMEN'I PFIRIOD
}

Orlakido M. Paiva

Assistente:

\author{
Armando Chibffi
}

Assistente, D.I.

Jỗo SOARES VHiga

I'rofessor

Ainda hoje são aceitos, de modo corrente, em observaçōes zootécnicas, ao se efetuar a determinação aproximativa da idade dos eqüinos, mediante exame dos dentes. ensinamentos colhidos em eras remotas e transmitidos até nossos dias sem a devida análise de exatidão. Como lemos em Connevin e Lesbre (1894), a avaliação da idade do cavalo já ao tempo de Xenofonte (400 A.C.) se realizava por intermédio dos chamados dentes indicadores - gnômonos (de conhecer, julgar). Aristóteles fornecendo-nos, em síntese, os conhecimentos da época sôbre o problema da idade dos eqüinos. meio a apreciações corretas e errôneas. enurciava os seguintes conceitos: aos 30 meses dá-se a perda dos quatro primeiros dentes (pinças); um ano após. ou seja. aos 42 meses, ocorre a queda de quatro outros (médios) e, superado idêntico intervalo, por outros têrmos, aos 54 meses, opera-se a substituição dos quairo dentes seguintes (cantos). Atribuía-se então capital importância - e esta noção ć, na atualidade, perfeitamente sustentável - ao período das mudas, durante o qual seria possivel estabelccer-se, com relativa segurança, a estimativa da idade.

Tivemos oportunidade de referir e de demonstrar, em estudo a propósito da cronologia dentária do zebu (Chisfri, PANA e VeIGa - 1918), que a generalização do emprêgo de índices em meios e em raças diversos é, reconhecidamente, prática falha. Os valores médios de avaliaçūo, assentados em base estatística, devem ter aplicação limitada às populações submetidas a estude.

Fstes fatos, aliados à absoluta escassez de dados bibliográficos firmados dentro das citadas normas, impeliram-nos a cumprir o presente trabalho, no Puro

(*) Aprescntado na IX Conferência Inual de Medicina Veterinária, realizada em Sho Prulo de 1 a 5 de dexembro de 1954. 
Tangue Inglès, sendo de realçar-se, antecipadamente, que os informes divulgudo* pelos tratadistas estão longe de corresponder à realidade, como provam as con. elusöes por nós inferidas.

\section{MATERIAI， F， MA'TODO}

Relacionamos, para investigação, 356 equinos puro sangue de carreira, de aiobos os sexos, mantidos em boxes individuais na Vila Hipica do Jockey Cluh de São Paulo.

Tendo fixado, posteriormente, limites para a leitura da idade, a consulta do Stud Book daquela organização impôs-nos o afastamento de 82 animais que, por ce situarem aquém ou além dêles, não se prestavam para estudo. Contamos. por isso, com 274 equiinos, a saher, 147 machos e 127 fêmeas, lodos em plens bida turfística ou em preparo para atingir o "estado".

0 exame foi realizado por um de nos, de maneira a abonar maior unifor. inidade na apreciação dos dados. Seguiu-se, para tanto. a técnica de rotina do observação direta das arcadas incisivas.

A esta altura seria oportuno chamar it atenção para os casos de permanéoria dos incisivos de leite, cuja presença, freqüente, näo alterava a leitura da idade, fundada na verificação dos dentes definitivos.

Enfeixamos os valores cbtidos em quatro grupos, assim designados: sem muda, primeira muda. segunda muda e terceira muda.

No primeiro grupo, ou seja, o dos animais sem muda, incluimos exemplares nos quais se encontravam à vista todos os dentes incisivos de leite, tanto superiores quanto inferiores. No segundo grupo foram congregados aquêles que mostravam a erupção de, pelo menos, uma das pinças definitivas. Do tercuiro grupo participavam os que, examinados, revelavam a existência de, no minimo. um dos médios permanentes. Por fim, enquadramos no último grupo espécime. cuja arcada incisiva nos permitia evidenciássemos, quando menos, urn dos caritos de substituição.

Distribuindo os 274 equiinos pelos mencionados grupos, obtivemos a seguinte relação:

a) sem muda - 68 animais, compres:ıdendo 36 machos e 32 fêmeas:

b) primeira muda - 86 exemplares, islo é, 41 machos e 45 fềneas;

c) segunda muda - 77 espécimes, dos quais 42 machos e 35 fêmeas;

d) terceira muda - 4.3 eqüinos, abrangendo 28 machos e 15 fêmeas. 


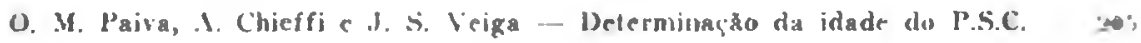

() exame do Stud Book, além de nos impor, como ficou dito, a exclusãu de 82 animais. possibilitou.nos a averiguação da idade real daqueles julgados em condiçöes de exame. O cálculo foi efctuado tomando-se por basc mês de 30.1 dias.

() dados catalogados foram submetidos a estudo estatístico, que constou da determiuaçāo da média. do êrro padrão, do êrro da média e do "t teste". Fista averiguação limitou-se aos grupos de animais em muda. 'Tendo estabclecido, preliminarmente. em separado, as médias e respectivos erros para machos e fêmeas. e mais, tendo verificado que as diferenças apuradas erain deslituidas de signifi. caçāo estatistica. computamos conjuntamente tôdas as variáveis.

Com o ohjetivo de facilitar a identificacão do número de dentes permanen. tes, nas dircrsas fases das mudas, resolveruos adotar representaçāo de tinada a fornecer. de relance, o tolal de substiluiçūes, valendo-nos. para isso. de fraçáo ordinária, cujo numerador e desominador especificavam, respeciivamente. os incisivos slipeniures e inferiores. Fxemplificando: a animal que trocara, diganos. sontente uma pinça superior, atribuiamos a fração $1 / 0$; a espécime que completara a speganda muda. conferiamos a indicação $4 / 4$; enfim, a eqüino que exihira unicamente un canto inferior de leite, Esziamos corresponder a notaçäo $6 / 5$.

\section{RESTLIAADOS}

Dentre 68 exemplares considerados no grupo dos sem muda. tomando-se 25 meses como limite minimo para a leitura, verificamos que 9 dêles $(13.2 \%)$ exibiam aquela idade, embora contassem menos de 26 mescs; 10.5 (15.5\%) distri. huiam-se entre 26 e 27 meses; 16.5 (24.3\% tinham mais de 27 e menos de 28 meses, enquanto 11 outros (16.2\%) repartiam-se entre 28 e 29 meses. (15 restantes 21 ofereciam a seguinte discriminação: $10(14.7 \%$ ) superavam os 29 , mas eslavam aquém dos 29.5 meses; 5 (7.3\%) ultrapassavam êste limile, porém não alingiam os 30 meses e os 6 últimos $(8.8 \%$ ) superavam já os 30 meses. Nesie lote incluimos 1 animal com 30 mescri e 17 dias.

() limite adotado para esta classe (25 meses) encontra sua justificativa no fato de que 1 dos exemplares nela enquadrados registrava apenas 25 meses e 9 dias de idade.

Para a primeira muda (Quadio 1) a média encontrada revelou-se igual a $1109.3 \pm 16.5$ dias, ou seja. 36.49 meses $(3$ anos e 14.9 dias). A distribuição dos valures dêste grupo estendia-se dos 25.3 (limite minimo) aos 42.3 meses (limite máximo). Anotamos a mais elevada frequîência na classe de 1150 a 1250 dias (30 observaçōes), devendo destacar-se que, à exclusāo de 1 animal - Ver nuth mostrava ainda uma das pinças inferiores de leite $(2 / 1)$ - nos mas exemplares as qualro pinças definitivas haviam surgido $(2 / 2)$. 


\begin{tabular}{|c|c|c|c|c|c|c|}
\hline & \multicolumn{3}{|c|}{$N .^{\bullet}$ de observaçōes } & \multicolumn{3}{|c|}{ Idade no período das mudas } \\
\hline & $\mathbf{M}$ & $\mathbf{F}$ & $\mathbf{T}$ & Dias & Meses & $\begin{array}{c}\text { Anos, mesed } \\
\text { e dlias }\end{array}$ \\
\hline Sem muda ..... & 36 & 32 & 68 & & & \\
\hline Primeira muda .. & 41 & 45 & 86 & $1109.3 \pm 16.5$ & 36.49 & 3a. $14.9 \mathrm{~d}$ \\
\hline Segunda muda... & 42 & 35 & 77 & $1417.3 \pm 19.5$ & 46.62 & $3 \mathrm{a} .10 \mathrm{~m} .18 .9 \mathrm{~d}$. \\
\hline \multirow[t]{2}{*}{ Torceira muda ... } & 28 & 15 & 43 & $1626.0 \pm 12.6$ & 53.49 & $4 \mathrm{a} \cdot 5 \mathrm{~m} \cdot 14.5 \mathrm{~d}$ \\
\hline & 147 & 127 & 274 & & & \\
\hline
\end{tabular}

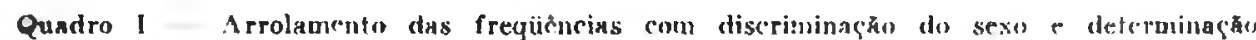
da idade dos anibiais no período das mudas.

Para que se tenha idéia da seqüiência rle erupção das quatro pinças perma. mentes (Quadro II) podemos aditar os seguintes esclarecimentos:

a) entre 750 e 850 dias, à exceção de 1 caso (Nha Iinda), no qual ape. nas uma pinça era visivel $(1 / 0)$, em quase tôdas as observaçóes estavam pre. sentes duas pinças superiores $(2 / 0)$; de outra parte, em l exemplar (Burma) restava por substituir sòmente uma pinça inferior $(2 / 1)$;

b) entre 850 e 950 dias, dos 13 espécimes examinados, 8 achavam-se na mesma situação da maioria dos animais reunidos no grupo precedente, isto é. denotavam a troca das duas pinças superiores $(2 / 0)$; os 5 restantes exibiam as quatro definitivas $(2 / 2)$;

\begin{tabular}{|c|c|c|c|c|c|c|c|c|c|}
\hline \multirow{2}{*}{ Diss } & \multirow{2}{*}{ N.• } & \multicolumn{2}{|c|}{$1 / 0$} & \multicolumn{2}{|c|}{$2 / 0$} & \multicolumn{2}{|c|}{$2: 1$} & \multicolumn{2}{|c|}{212} \\
\hline & & n..$^{\circ}$ & $\%$ & $\mathrm{n} .^{2}$ & $\%$ & $n \cdot$ & $\%$ & $n 0^{\circ}$ & $\%$ \\
\hline $750-850$ & 6.0 & 1.0 & 16.7 & 4.0 & 66.6 & 1.0 & $16 . i$ & - & - \\
\hline $850-950$ & 13.0 & - & - & 8.0 & 61.6 & - & - & 5.0 & $3 S .4$ \\
\hline $950-1050$ & 8.0 & - & - & - & - & 1.0 & 12.5 & 7.0 & 87.5 \\
\hline $1050-1150$ & 14.0 & - & - & - & - & - & - & 14.0 & 100.0 \\
\hline $1150-1250$ & 30.0 & - & - & - & - & 1.0 & 3.4 & 29.0 & 96.6 \\
\hline $1250-1350$ & 15.0 & & - & - & - & - & - & 15.0 & 100.0 \\
\hline & 86.0 & 1.0 & 1.2 & 12.0 & 13.9 & 3.0 & 3.5 & 70.0 & 81.4 \\
\hline
\end{tabular}

Quadro II - Representaçăo das frequências e respertivas porcentagens, na primeira unda, de acoido com on nimero de pinças irrompidas. 
c) rntre 950 e 1050 dias e nas classes subsequientes, excetuando-se 2 exemplare:- Donga c Vermuth ainda exibiani uma das pinças inferiores de leite 12/1) - codos os outros linham completaio a muda (2/2).

En termos modios. $x$ segunda muda (Quadro 1 ) foi fixala em $1117.3+19.5$ dias, ou seja. 16.02 meses 13 anos. 10 meses e 18.9 diasl. Os valores calalo. gados dispunham-se entre os 37.5 e 54.3 meses, têmos, respectivamente, mínimn r máximu. Diras das classes englohavam " mesmo e maix elevado número de frequîencias. ou seja, 22: as que sc situavam entre 1100 e 1700 dias.

Passemos à análise dêsle grupo (Quadro III), pormenorizando a compo. siçä́o de cada tlasse em separado:

21 chre 1100 e 1250 dias compulamos is espécimes com apenas um médie superior $(3 / 2)+1$ outro com um úmico midio inferior permanente $12 / 3)$; reubimos 5 exemplares denotando a presença de seis dentes definitivos, sendo que l mostrara um médio em cada arcada $(3 ; \%)$; 1, dois médios na arcada supe. rior $(1 / 2)$ o os 3 segulintes somente os inferiores $(2 / 4)$; registramos, ainda: 2 exemplare: nos quais ś restava por trocat um médio inferior de leite $(1 / 3)$; 2 animais com persistência de um inico médio superior caduco (3/4) e, pur fim. 6 efüinos com oito destes refinitives $(4 / 4)$ :

1) enire 1250 e 1100 dias verificanos $l$ caso de permanência de três mé dion temporários, dois superiores e um inferior $(2 / 3) ; 7$ casos de queda the dois médios decíaluos, nolando-se 1 vez a presenca dos inferioces $(4 / 2)$ vêzes a dos suleriores $(2 / 1) ; 1$ exemplar com apenas un médio superior de leile $(3 / 4)$ c, por ultimo, 5 animais com a muda concluida $(1 / 1)$;

\begin{tabular}{|c|c|c|c|c|c|c|c|c|c|c|c|c|c|c|c|c|c|}
\hline \multirow{2}{*}{ Dix: } & \multirow{2}{*}{$\therefore$} & \multicolumn{2}{|c|}{$3 \% 2$} & \multicolumn{2}{|c|}{$2 ; 3$} & \multicolumn{2}{|c|}{$3: 3$} & \multicolumn{2}{|c|}{$4_{i} 2$} & \multicolumn{2}{|c|}{$2 ; 1$} & \multicolumn{2}{|c|}{$4 ?$} & \multicolumn{2}{|c|}{$3+$} & \multicolumn{2}{|c|}{$t ; 1$} \\
\hline & & $11^{\circ}$ & $\%$ & $11^{n}$ & $\because n$ & $11 \cdot$ & $\%$ & n. ${ }^{\circ}$ & 111 & & io & n. & $\%$ & 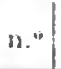 & $\because n$ & $u^{\circ}$ & \% \\
\hline $100-1250$ & 19.0 & 30 & $15 . \mathrm{s}$ & 1.0 & 5.3 & 1.0 & 5.3 & 1.0 & 5.3 & 3.0 & 15.8 & 2.0 & 10.5 & 2.0 & 10.5 & i. 0 & 315 \\
\hline $250-1300$ & i110 & - & -1 & 1.0 & 7.1 & $\cdots$ & -1 & 1.0 & 7.1 & 6.0 & +2.9 & - & -1 & 1.0 & 7.1 & 5.0 & 35 \\
\hline $400-1550$ & 22.0 & - & - & - & - & -1 & - & - & -1 & - & - & - & -1 & & -1 & 22.0 & 1000 \\
\hline $550-1700$ & 22.0 & 1.0 & 1.5 & - & -1 & - & -- & - & - & 1.0 & 4.5 & - & -1 & - & - & 20.0 & 91.0 \\
\hline & 8.0 & 4.0 & 5.2 & 2.0 & $2.6 i$ & 1.0 & 1.3 & 2.0 & 2.6 & 10.0 & 13.0 & 2.0 & 2.6 & 3.0 & 3.9 & 53.0 & 03. \\
\hline
\end{tabular}

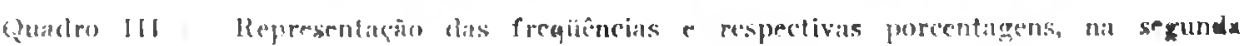

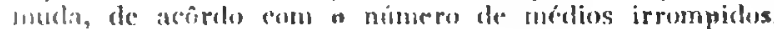

(1) as classes inscritas entre $1.400 \mathrm{e} 1700$ dias pudern ser consideradas con. junlamente porquanto nelas se enzquadravan exemplares que, salvas 2 observa-

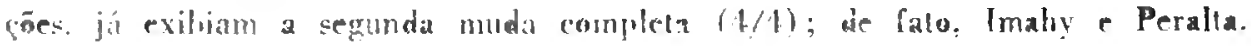


alistalos na classe de 1550 a 1700$)$ dias. :mostravan, respertisamente. a subsil luiçäo de apenas un dos médios superiores $(3 / 2)$ e a conservaçäo dos dois mé dios aprerioures caducos (2/1).

Tal como lizemos para o grupn dos animais sem muda, fixamos. tamberm. limile máximo para leilura da mesa denlárix. Como. de lima parte, a- labelan clissicas extabelecem para at terceira muda " praso de 00 meses r como. por outro lado. não ascinalamos um caso sequer de pernancincia de rantos tempora riox en animais com idate superior àquela. arbilramos em 1824 din: $(6)$ mese: nax ba:e de 30. ! dia:1 "indice exiremo para collheita de dado:

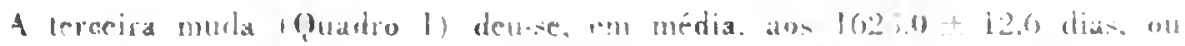

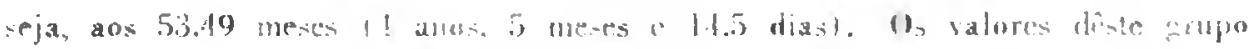

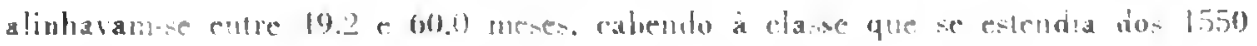

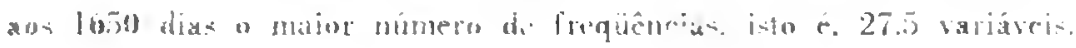

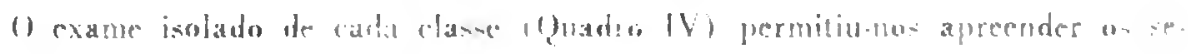
suinles dasins adirionain:

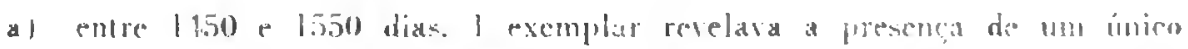

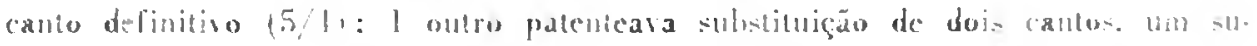
perior e u:n inferior $(5 / 5)$; os 1.5 schuintes havian trocado simente os raitos

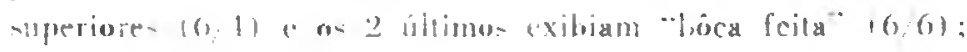

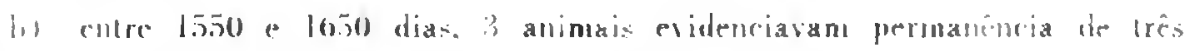

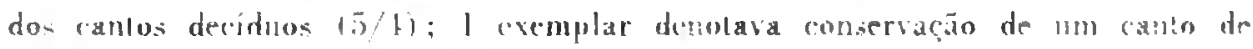
leile en cada umat das areadas 15/51; em 12.5 observacues positivamos a exis. lencia de doik canlos definilios, quer superiores 16/1). como vimus em 9.5 ca

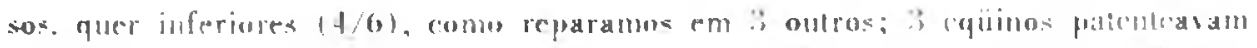
a coistencia de um inico canto inferior caluco $16 \%$; mais com subilituigäe lonal dos incisivos (0,6);

\begin{tabular}{|c|c|c|c|c|c|c|c|c|c|c|c|c|c|}
\hline \multirow{2}{*}{ Dinz } & \multirow{2}{*}{$x$} & \multicolumn{2}{|c|}{54} & \multicolumn{2}{|c|}{55} & \multicolumn{2}{|c|}{ b. 1} & \multicolumn{2}{|c|}{46} & \multicolumn{2}{|c|}{ (i) i) } & \multicolumn{2}{|c|}{ iiil } \\
\hline & & $1 .^{\circ}$ & $\%$ & $\mathrm{t}^{*}$. & $n$ & $u^{\circ}$ & $\because$ & $110^{\circ}$ & $o_{0}$ & $n *^{*}$ & ${ }^{\circ} n$ & $11 .{ }^{n}$ & $\%$ \\
\hline $1400-15: 00$ & 5.5 & 1.11 & $1 \leq .2$ & 1.0 & $1 \leqslant 2$ & 1.5 & 27.3 & - & - & - & - & 20 & 36. \\
\hline $1550-165$ & 27.5 & 3.0 & 10.9 & 1.0 & 3.6 & 9.5 & 34.6 & 3.0 & 10.9 & 3.0 & 10.4 & $\$ 0$ & 24.1 \\
\hline $1650-1750$ & +0 & & - & - & - & 1.0 & 25.0 & - & - & 1.0 & 25.1 & 2.0 & ill. \\
\hline 175018511 & 6.11 & & - & 111 & $11 i .7$ & - & - & - & -- & & $\longrightarrow$ & 5.0 & s;:; \\
\hline & 43.0 & 4.0 & 9.8 & 3.0 & 7.0 & 12.0 & 27.9 & 3.0 & 7.0 & 9.0 & $9 ! 3$ & 1.60 & 39.5 \\
\hline
\end{tabular}

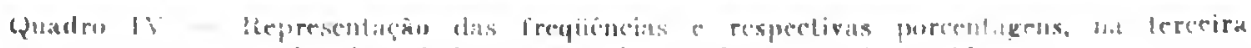

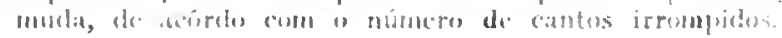




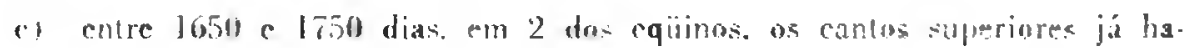
tiam jernmpido (6/1), enduanto, na mandíbula aperas aparecia, cn I dos ani. mais. un dos camos definitivos $(6 / 5)$; nos 2 reslantes podiam ser apreciados 0 se seis incisivos permanentes $(6 / 6)$;

d) entre 1750 o 18,50 dias, exceluando-se 1 espécime lliaralhoi, an qual anda nobivanos a existencia de dois cantos de leite, um superior e um inferior $1.5 .5)$, non mais a periodo das mudas cstaina tominado $(6 / 6)$.

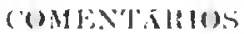

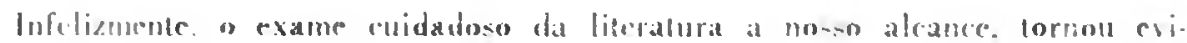
Iente. romo afirmames: a falla completa de: datus colalisticos a propósilo da

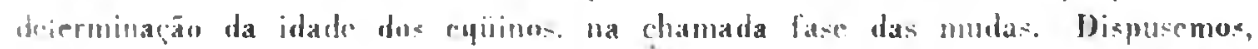

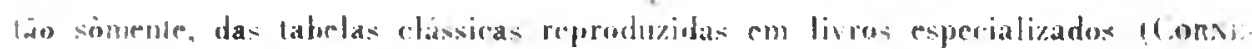

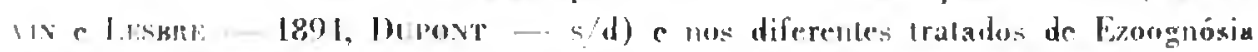

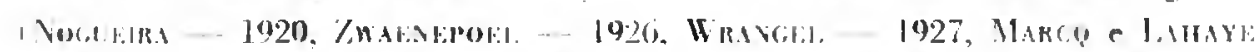
10:3, Vordive
19:37, Ms(i.11 vo
195i), J J trims
1952 e Kiाs
19.5.3).

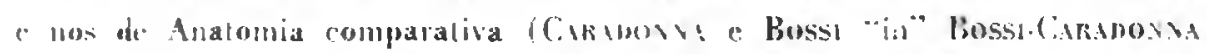

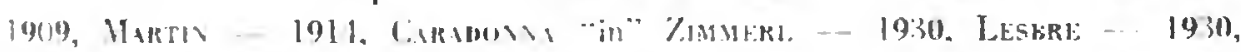

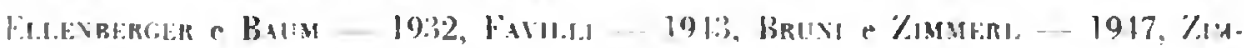

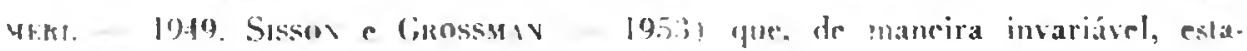
tueren as aceruintes dados:

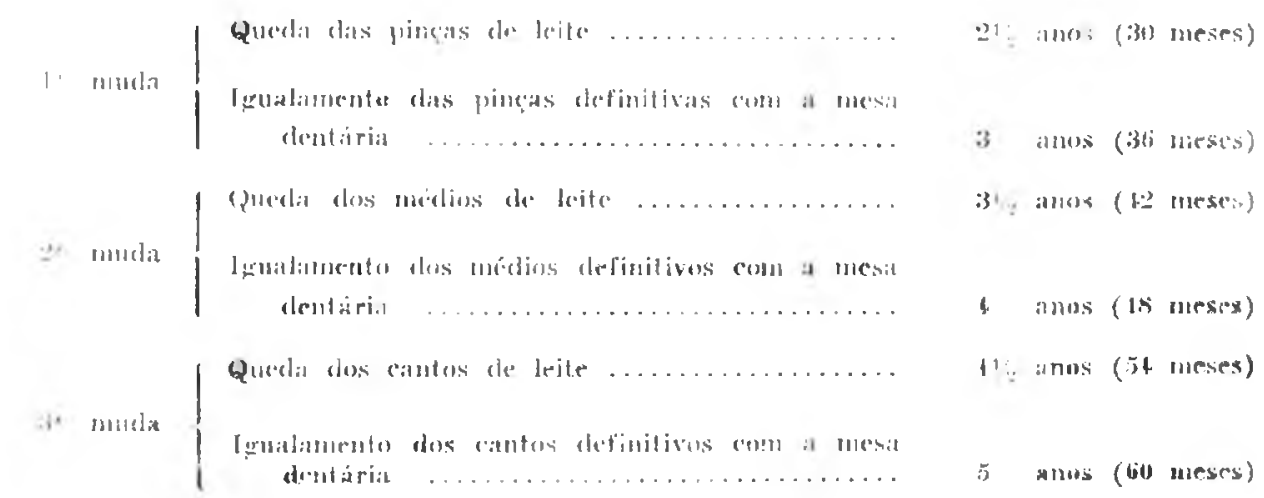

De aroirdo com os resullados ascontanios, a primeira muda processar-se-ia ans 30.19 meses, a segunda aos 16.62 mesps a terceira ans 53.19 meses. Ora. considerandes-se a repartiçấo dos animais :m strupos, nos quais alistamos tanto as exemplares com a muda apenas em inicio quanto aquêles que acabavam de completäla, não nos parece fora de propusicu o estudo comparativo entre os va- 
lores médios por nus ralculados e as nédias aritmélicas des indires aproutarupara cada substituiçä́n pelos tratadistas, cucaradas como expressão unilúria dafases. Concosnte tal critério, a primeira muda oromeria aos 3.3 mexpes. a se sunda aos 45 e a terceira gos 57 ineses. A romparaçio, como se icrifica now Quadro V, permite-nos al gumas ponderaçöes, "mhora forçuso seja reconhecur nīi tenham a expressäo desejácel $c$ atimgivel a ionmuladas fonsem im têrmos esti. lislicos.

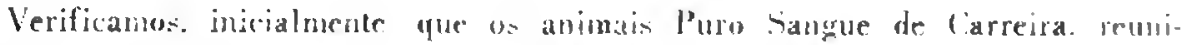
dox para a pesquisa, efetuavam a sigunda muda aos f6.62 meses, enquanto săn

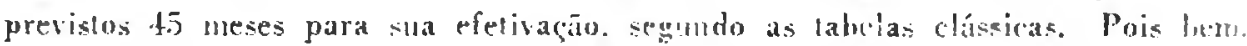
talve\% sejam fistes os únicos dados para os quais, provavelmente, a diferenga to balística se desvectirá de significarän. De falu. ohservam-se, para a primeira r ultima middas. marcadas discordancias. por (e)incidencia da ordem de 3..5 me. ses, de morto que o Puro Sangue ligwlis. enil noson meio. enceta o periodo de nilidas con atrazo sobre os índices estabelechlos e completa a stbbstituição tutal

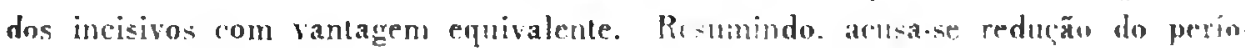
do das mudas de 21 para 17 meses.

\begin{tabular}{|c|c|c|c|c|c|}
\hline \multirow[t]{2}{*}{ II $\mathrm{das}$} & \multicolumn{2}{|c|}{ Tratsdistas } & \multicolumn{2}{|c|}{ Ubservasóns los AA. } & \multirow{2}{*}{ 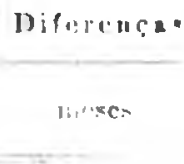 } \\
\hline & $\begin{array}{c}\text { Variacinon } \\
\text { mescs }\end{array}$ & $\begin{array}{l}\text { Média } \\
\text { meses }\end{array}$ & $\begin{array}{l}\text { Variagaso } \\
\text { mexess }\end{array}$ & $\begin{array}{l}\text { Media } \\
\text { mexes }\end{array}$ & \\
\hline P'rimeir:t musa. & $30-36$ & $3: 3$ & $25.3-423$ & 36.49 & $: \quad 3.49$ \\
\hline Siguncla muda. & $12-4 s$ & 15 & $37.5-54: 3$ & 11i.62 & 1.62 \\
\hline lereceira muda & 5t - hil & $\pi$ & 49.2 & 53.49 & 351 \\
\hline
\end{tabular}

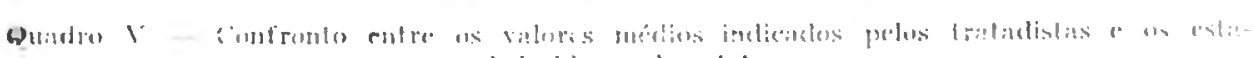

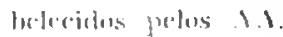

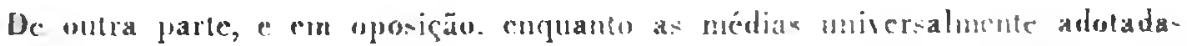
reclam estranha uniformidade para o inievalo das nudas 112 meses, já non moso material comprobamos assinalada desigualdade a espaçílas. Acsim. -r

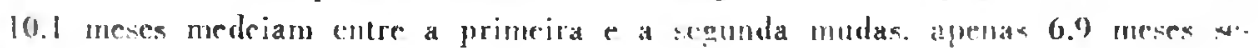
yaraia esiu úllima da tercerira.

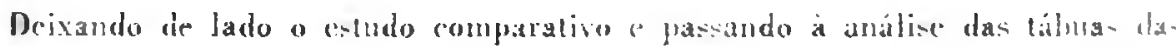
freqüencias (Quadros II, III e IV), nas quais discriminamos a orden dil lrua dos incisiros, uberramos que:

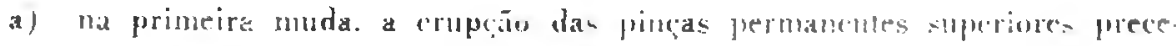
dr: le forma consante. a das inferiores: 
b) na secunda mudx, inversamente, médios definitiven inferieres, com maior freqüência, anteorem a aparecinento dos correspondentes superiores is cacos em 23);

c) na lerceira muda, aparecimente dos ranle: inferiores sucede, quase sempre, 2o dos superiores (20 casos em 23$)$;

d) a troca de pingas (4 casoe), médios (12 obrervaçors) e cantos (11 variavejs) nä ocorre, wuitas vêzes, simulláneantente. nas duas liemiarcadas; rm alsun exempiaces (4) dos grupos de 12 e 11 animais, enquadrados. rexpectivamente. nos periober da cegunda (l) e terceira 13) madas. esla característica atinge alulias as arcadas.

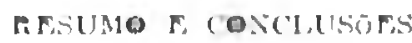

Visando estabelecer. ean base estatistica, meios para determinacia da idade

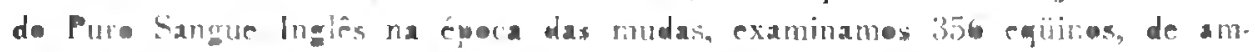
bor os sexws, mantidos em "boxes" individuaik na Vila Hipica do Jockey Ciub de Sĩ. Paule.

Llininados os animais (\$2) que näo se prestavam ao pretendido estude, conpulamos 27. exemplares (147 machos a 127 fêncas), os quais, de conformidade com o exame direto das arcadas incicivas, foram distribuidos em quatro grupos: a) sem inuda - $6 \%$ animais (36 machos e 32 fêmeas); b) prineira muda - 86 exesuplares (41 machas e 45 fêmeas); c) segunda nuda - 77 equinos (12 machos e 35 fêmeas); d) terceira muda - 43 expécines (28 machos e 15 fêneas).

A daid de nascimento inscrita no Stud Book mantido pelo Jockey Club permitiu-nos o cálculo da idade real, efetuado na base de 30.4 dias por mês.

A interpretação eslatística, limilada aos grupos de animais em neuda, levounos a arrolar as variáveis. englobadanente, pôslo que denunciamos náo signi. ficantes as diferengas eucontradas entre os dois sexos.

Adotamos representação destinada a fornecer, de inediato, o ninuero de dentes substituidos, mediante o emprêgo de fraçäo ordinária, cujo numerador e denominader nos davam, na ordem de enumeraçäo, o total de incisives superiora e inferieres permanentes.

Face aos resultados obtidos. parece-nos licito firmar as seguintes couclusóes:

a) convencionando-se como ponte de partida, para a leitura, es 25 mesea de idade, dentre 68 animais do grupo dos sem muda, 9 (13.2\%) linhaw meno de 26 meses, $10.5(15.5 \%)$ estavam aquém dos 27 maeses, 16.5 (24.i\%) mäo 
haviam completado 28 meses, enquanto 11 outros $(16.2 \%$ c) repartian-sc entre 24 e 29 meses; dos restantes, 10 (11.7\% c c) ("nntisam mais de 29 e menos de 29.3 meses; $5(7.3 \%)$ distribuiam-se cutre: 29.5 e 30 muses e $6(8.8 \%$ c) superavan jai os 30 meses;

b) a primeira muda dí-ce, cm têrmos médios, aos 1119.3 - 16.5 dias, ou seja, aos 36.19 meses (3) anos e 11.9 dias) ;

c) a substituigão dos médios de leite ororre, cm média, alos $1117.3 \pm 19.5$ dias, isto 2 , aos 16.62 meses $(3$ anos, 10 meses o 18.9 dias);

d) a terceira muda processa-se, nas mesmas condiçoes, aos $1626.0 \pm 12.6$ dias. por outras palavras, aos 5.3 .49 meses (1 anos, 5 meses e 11.5 dias);

e) o estudo comparativo entre os dados por mós assentados e os valorrs midios consignacios em tratados de faogonisia e de Allatomia comparativa fa\%. nos crer que o l'uro Sangue de (arreira, cm nosso meio, inicial ') periodo de mudas cont alraso de 3.5 meses sôhre os indices divulgados e exile "hôra feita" con rantagem equivalente, adotando-se ainda for hase os citados valores;

f) hit apreciácl reduça do periodo das substitucẹes, cumpribo irregularniente em 17 meses, pois, se o intervalo ente a primeira e segunda mudas al. cança 10.1 meses, ja o que medeia entre "-sa e a derrulcira restringe-se a 6.9 moses;

g) lla primeira e terceira mudas, à excecão de thés casos relativos à úlima dclas, a erupgiáo, respectivamente, de pinçes e cantos superiores precede a dos dentes homônimos inferiores; em contrapartiria, os medios definitivos inferiores antecedem. na maioria das observaçoes, ao aparecimeno dos superiores;

h) o afloramento dos incisivos permancontes não sobrevém, de forma sistematica, simultäneanente, nas duas hemiarcardat, como patenteamos em 27 animais.

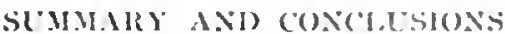

A statistical study is presented dealing with the average lime at which the replacement of the temporary incisor teeth by the permanent ones is performed in Thoroughbred kept under training conciitions at Jockey Cilul, of S. P'anlu, lirazil.

For this purpose, 356 horses were examined. from these, 82 were excludud and the remainders 27.4 (147 males and 127 females) disided in four grouss in accordance with the eruption of their incisor teeth: a) without permanent incisors - 68 horses (30 males and 32 remales); h) with ciller one, two, thre or four permanent pincers - 36 horses 11 ! maks anc. 15 females); c) with all the permanent pincers, and cither one, wo, three cot fout permat 
(). M. L'tira, A. Chieffi c.J. S. Veriga - Determinaçĩo da idlade do P.S.C.

nent intermediates 77 horses (12 males and 35 females); d) with all the fermarest pincers and infermediales and cither one, two, thece of four permanem

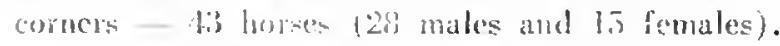

The actual age of each horse was controlled by the siarl look recoris; in.: dags was the period coubsidered as mosith average.

Sex differences showed! mo statistical significalion at age for treth reularement. For this reason males and females were computed all togetiver.

The following points were olverved:

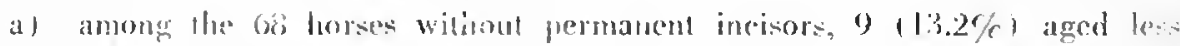

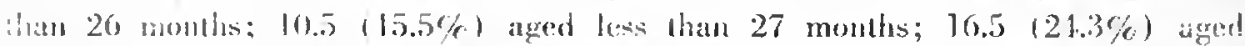

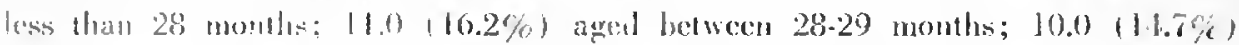

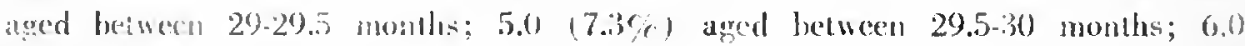
lis.8\%? aged mose thatn ino monils:

b) permanent pincers eruption was serified in horses aberaging 1109.3 i \pm 16.5 days of age $1: 3$ years and $1: 49$ days);

c) permanent internediates eruption was noted in horses averaging 1117.: 19.5 days of age (3 years, 10 months and 18.9 days);

d) permanent comers eruption was stated in horses avcraging 1626.0 上 12.6 days of age (1 years, 5 months and 11.5 days);

p) it is pointed out from these studiss that the Thoroughied animatio raised in S. Thalo State start incisors replacement 3.5 monthe later than indieated hy classical tables: on the other hand, they have all incisors repiaced 3.5 months earlier than the data foresen in the classical lables;

f) the eruption of permanent intermediates leok place 10.1 months in aver.

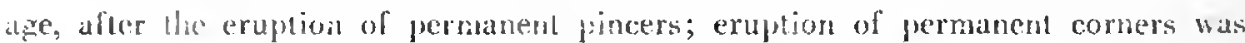
observed 6.9 months in average, alter the rruplion of permanent internediates:

(x) the eruption of the superior pincers and corner tech occured cartier in relation to the inferior ones in all cases out of three; most conmonly the inferior permanent intermediaies come out before the supror bass;

h) in 27 animals the permanent incisors did not appear on rach areade: at the same limes.

\section{H3HBLIOGIRAFIA}

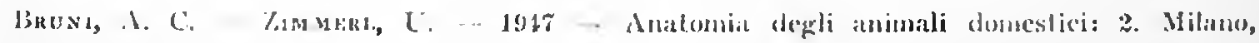
Franceseov Vultardi 
Caramonsa, G, 13. - Bossi, V. - 1909 (pref.) - "in" IBossi-Camanonsa - Iratlato di Anatomia Veterinaria: 2. Milano, Francesco Vallardi

Camadoxsa, (i. 13. - 1930 - "in" Yimmers, U. - Trattato di Anatomia Veterinaria: D. Milano, Francesco Vallardi

Chiefri, A. - PNiva, O. M. - Viiga, J. S. - 1918 - Contribuiçĩo para o estude da cronologia dentária no licbu. Mez. Fac. Med. l’et., S. l’aulo, 3(1):251/69

Connevis, C. - Iesme, F. X. - 1894 - Traté de liage deg animaux domestiques d'après les dents et les productions épidermidgues. 1'aris, J. I. Bailliere et fils

Duront, M. - s/d. - I'age du cheral et des principaux animax donestiques. l'aris, J. I3. I3aillière et fils

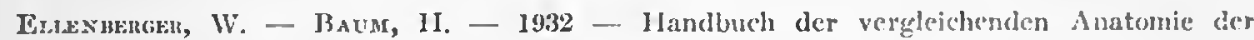
Haustiere, 17 Aufl. Berlin, Julius Springer

FavıL., N. - 1913 - Noxioni comparate di Anatomia c risiologia degli animali agricoli. Milano, Francesco Vallitrdi

Jarumar, W. IR. - 1952 - Exterior e Julgamento dos Liepuideos (Cavalos, jumentos . muares). 2* cd. melhorada. I’iracicaba, Tip. Jornal de I'iracicaba

Kaxs, D. J. - 1953 - The Iforse. Judgring, brecking, feeding, managenent, selling. New York, A. S. Barues \& Co.

Lesme, F. X. - 1930 - I'recis d'Exterieur du cheval ct des principaux mammifires domestiques. Jimo éd., l'aris, Vigot I'rìres

Magriano, A. - 1950 - Ezoognosia generale. Milano, Francesco Vallardi

Matca, J. - Lamaye, J. - 1934 - Extericur du cheval. Gemblaux, Jules Duculot

Marrix, P. - 1911 - Lehrbuch der Anatomie der Haustiere: 2(1). Stultgart, Schickhardt $\&$ Iibner

Noovema, O. R. - 1920 - Exterior dos grandes animais dunćsticos. São Paulo, Olegário IRibeiro.

Sisson, S. - Grossmas, J. D). - 1953 - Anatomia de los Animales Domesticos. 3* ed. Madricl, Salvat Editores $\mathrm{S} / \mathrm{A}$

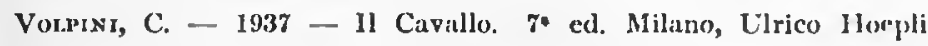

Wranoei, G. - 1927 - Dis 13uch vom Pferde: 1. Stuttgart, Schickhart \& Eloner

Zimmikr, U. - 1949 - Anatomia Iopografica Veterinaria. Milano, Irancesco Vallardi

Zwannepoei, K. - 1926 - Précis du cours d'Extéricur du Cheval. 2eme éd. Bruxclles, G. I3othy 